\title{
Leakage current effect on fixed and tunable solenoid RF MEMS inductors
}

\author{
Nizar Habbachi \\ Microelectronics and Instrumentation Laboratory, Faculty \\ of Sciences of Monastir, University of Monastir, 5000 \\ Monastir, Tunisia, \\ habbachinizar@yahoo.fr
}

Hatem Boussetta

Institut Prépratoire aux Etudes d’Ingénieurs de Monastir, Rue Ibn Eljazzar - 5019 Monastir Tunisia, and he is with Microelectronics and Instrumentation Laboratory, Faculty of Sciences of Monastir, University of Monastir, 5000

Monastir, Tunisia, hatem_boussetta@yahoo.fr

Mohamed Adel Kallala

Military Research Centre, Tunis, and he is with Microelectronics and Instrumentation Laboratory, Faculty of Sciences of Monastir, University of Monastir, 5000 Monastir, Tunisia, Adel.kallala@gmail.com

\author{
Ali Boukabache \\ Laboratory for Analysis and Architecture of Systems, \\ CNRS , LAAS, 7 avenue du Colonel Roche, 31077 \\ Toulouse, France, and he is with the University of Toulouse, \\ 1077 Toulouse, France, \\ aboukaba@laas.fr \\ Patrick Pons \\ Laboratory for Analysis and Architecture of Systems, \\ CNRS, LAAS, 7 avenue du Colonel Roche, 31077 \\ Toulouse, France, \\ ppons@laas.fr \\ Kamel Besbes \\ Center for Research on Microelectronics \& \\ Nanotechnology, CRMN Sousse TechnoPark, BP 334 \\ Sahloul Sousse, Tunisia, and he is with Microelectronics \\ and Instrumentation Laboratory, Faculty of Sciences of \\ Monastir, University of Monastir, 5000 Monastir, Tunisia, \\ kamel.besbes@fsm.rnu.tn
}

\begin{abstract}
This paper investigates the performances of fixed and tunable solenoid RF MEMS inductors for two silicon substrates: regular and etched. We have modeled the current density distribution in silicon substrates and then in NiFe plate using FEM numerical tool. Moreover, we have studied the inductors performances in responses of frequency variation from $100 \mathrm{MHz}$ to $30 \mathrm{GHz}$. The best quality factor and resonant frequency values are respectively $Q_{\max }=19$ and Fres $=15 \mathrm{GHz}$ when substrate leakage current is reduced. In addition, the obtained tuning range increases and reaches $\operatorname{Tr}=132.8 \%$ at $10 \mathrm{GHz}$ instead of $\operatorname{Tr}=96.8 \%$ at $5 \mathrm{GHz}$ when the silicon substrate is etched.
\end{abstract}

Keywords—RF MEMS; solenoid; inductor; tunable; microwave, eddy current.

\section{INTRODUCTION}

RF MEMS inductors have drawn much attention owing their versatility in microwave domain [1]. Several actuation techniques were proposed in order to ameliorate the RF MEMS devices performances [2-7]. In all contributions, RF designers need to increase the tuning range, the quality factor, and the resonant frequency. The most reported tunable RF MEMS inductors are based on spiral geometry modification [3-4]. An external actuation manipulator imbedded in the inductor center is used to control the core height [3]. The inductor performances are characterized at $2 \mathrm{GHz}$ for a total distance variation of $150 \mu \mathrm{m}$. The tuning range and the quality factor are respectively $\operatorname{Tr}=3 \%$ and $Q=2$. A more efficient tunable MEMS inductor using the dilatation of spirals turns under the joule effect is investigated in [4]. Indeed, the variation of spirals positions with respect to the substrate makes it possible to increase the quality factor and the resonance frequency. The characterization of this structure shows a tuning range of $\mathrm{Tr}=18 \%$ and a quality factor $\mathrm{Q}=13$ with a high frequency resonance Fres $=15 \mathrm{GHz}$.

In this paper, we present the effect of the leakage current on solenoid RF MEMS inductors performances: fixed and tunable. At the first part, we present our main structure with detailed dimensions in 3D perspective. Then, we investigate the current distribution in two cases of substrate: conventional and etched. At the second part, we discuss the displacement of ferromagnetic thin film inner solenoid core on inductor performances. The obtained results demonstrate the importance of etched substrate in the improvement of the response of our tunable inductor.

\section{RF MEMS FIXED INDCUTOR}

\section{A. Structure responses with regular substrate}

We have designed seven turns core using copper metal due to his high electrical conductivity $\sigma=57.10^{6} \mathrm{~S} / \mathrm{m}$. The substrate is a high resistive silicon $\sigma=3 \mathrm{~S} / \mathrm{m}$ and possess a relative permittivity $\varepsilon=11.2$. The Fig 1 illustrates the 3D perspective of our structure accompanied with detailed dimensions: 


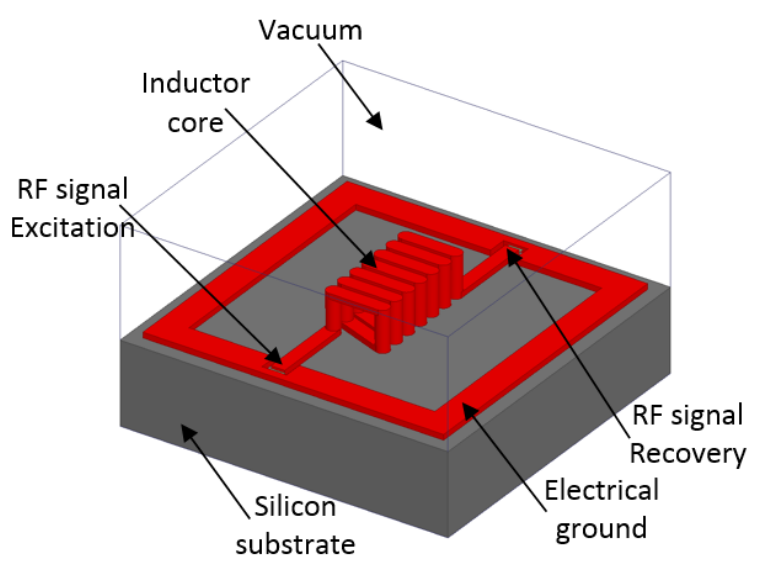

(a)

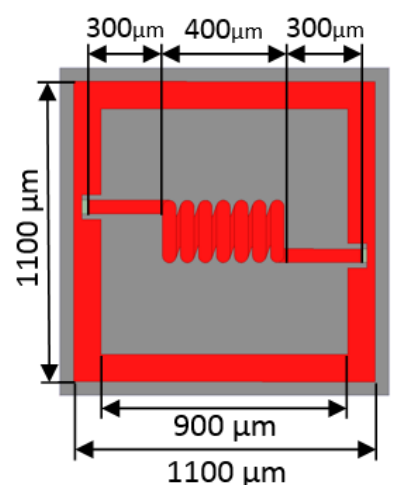

(b)

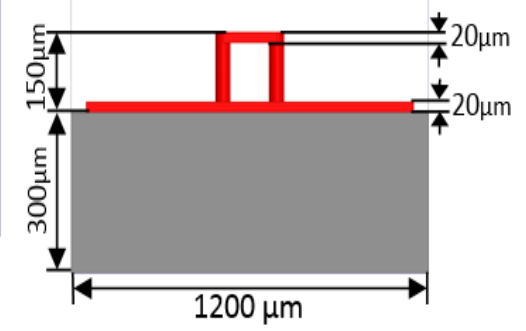

(c)

The response of RF inductors depends on geometrical dimension and electrical propriety of used materials. The inductance and the quality factor values are extracted from the Y parameters using the equations below:

$$
\begin{gathered}
\mathbf{L}(\mathbf{f})=\frac{\operatorname{Im}(1 / \mathrm{Y} 11)}{2 * \Pi * \mathbf{f}} \\
\mathbf{Q}(\mathbf{f})=\frac{\operatorname{Im}(\mathbf{Y 1 1})}{\operatorname{Re}(\mathbf{Y 1 1})}
\end{gathered}
$$

The Fig 2 presents the RF performances of our inductor for a frequency range from $100 \mathrm{MHz}$ to $30 \mathrm{GHz}$ :

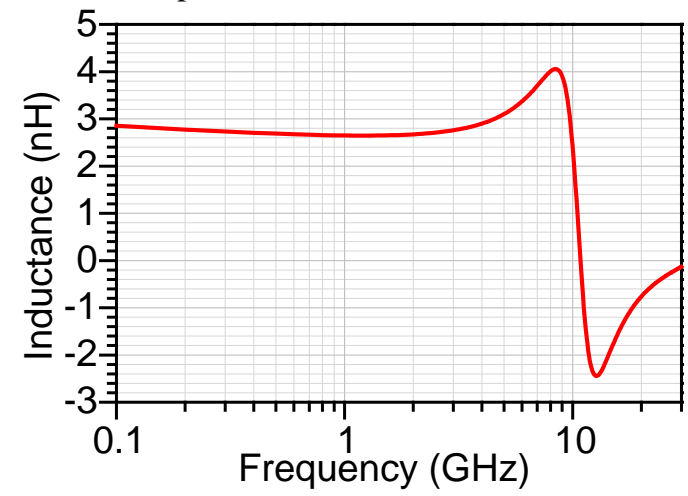

(a)

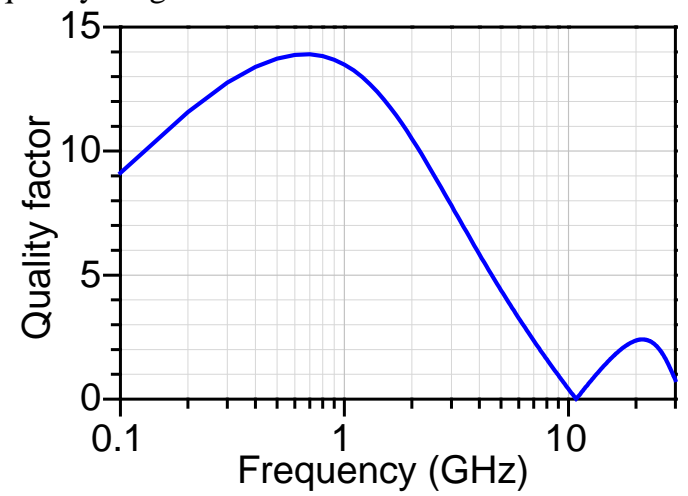

(b)

Fig. 2 Inductor performances in response of frequency variation (a) inductance value (b) quality factor.

The Fig 2.a shows a good stability of the inductance value until $8 \mathrm{GHz}$. Indeed, the inductance value is comprised between 2.8 $\mathrm{nH}$ and $4 \mathrm{nH}$ and the resonant frequency reaches $10 \mathrm{GHz}$. Moreover, the quality factor is greatly higher than spiral RF inductors [34]. The maximal value of the quality factor $Q_{\max }=14$ is obtained at $700 \mathrm{MHz}$. The performance of the RF inductor is also related to the substrate losses as the eddy current.

We have investigated the current distribution in the silicon substrate at $5 \mathrm{GHz}$ and in response of other frequencies: $100 \mathrm{MHz}$, $10 \mathrm{GHz}, 20 \mathrm{GHz}, 30 \mathrm{GHz}$.

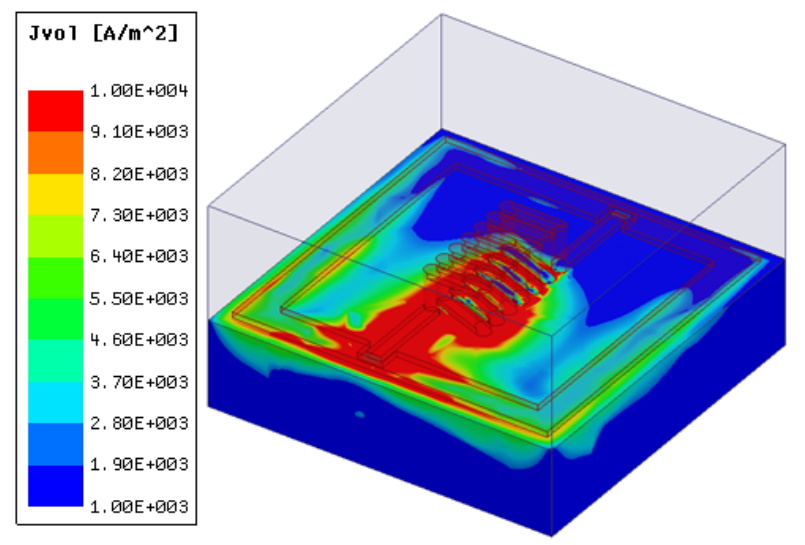

(a) 


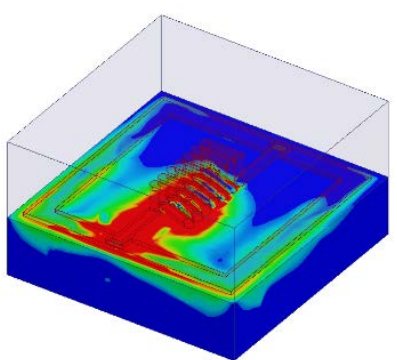

(b)

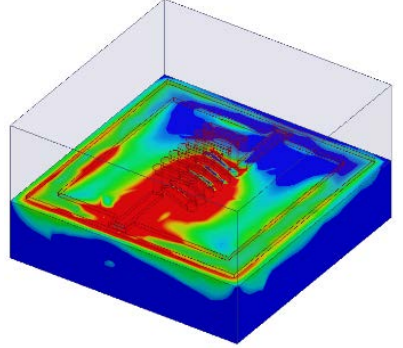

(d)

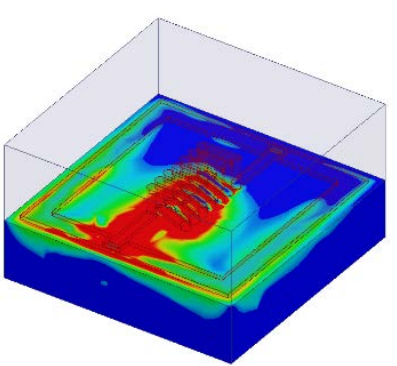

(c)

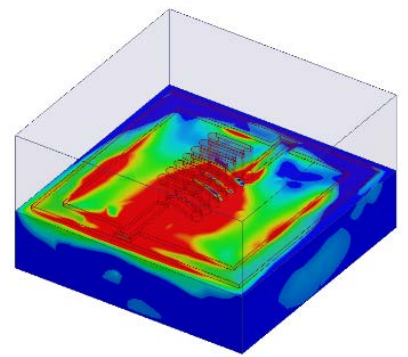

(e)

Fig. 3 Distribution of electrical current density in silicon substrate for different frequencies (a) aim frequency $5 \mathrm{GHz}$ (b) $100 \mathrm{MHz}$ (c) $10 \mathrm{GHz}$ (d) $20 \mathrm{GHz}$ (e) 30 $\mathrm{GHz}$.

As we can see in Fig 3.a, the maximum amount of distributed current is localized underneath the excitation port and the inductor's core. In addition, the substrate leakage current rises in response of frequency variation as illustrated in fig 3(b-e).

\section{B. Structure responses with etched substrate}

In the previous part, we have concluded that the silicon substrate is condemned by the excessive leakage current that could decrease the RF performances of our inductor. Therefore, we have etched the volume underneath the core of solenoid inductor as shown in Fig 4.

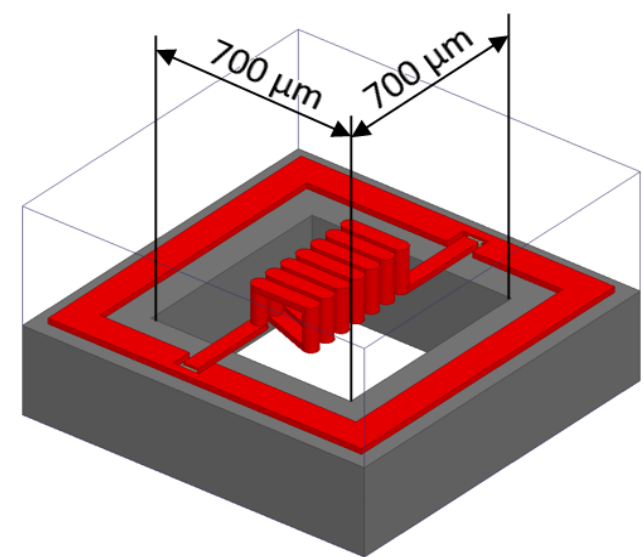

Fig.4 3D perspective of RF MEMS solenoid inductor structure with etched substrate.

The Fig 5 illustrates the RF performances of the new solenoid inductor in response of frequency variation from $100 \mathrm{MHz}$ to 30 GHz:

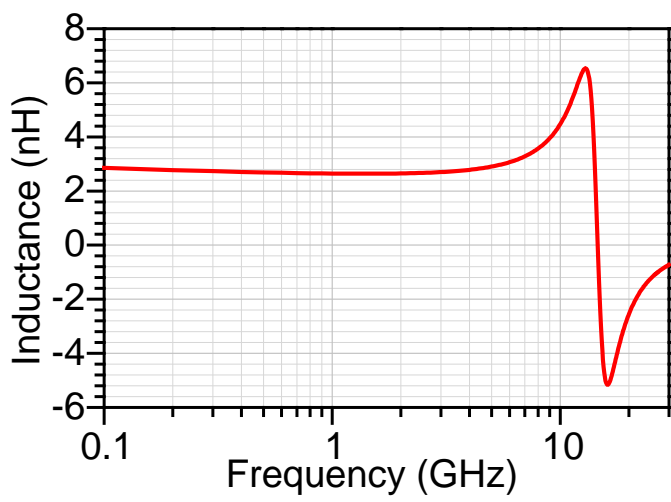

(a)

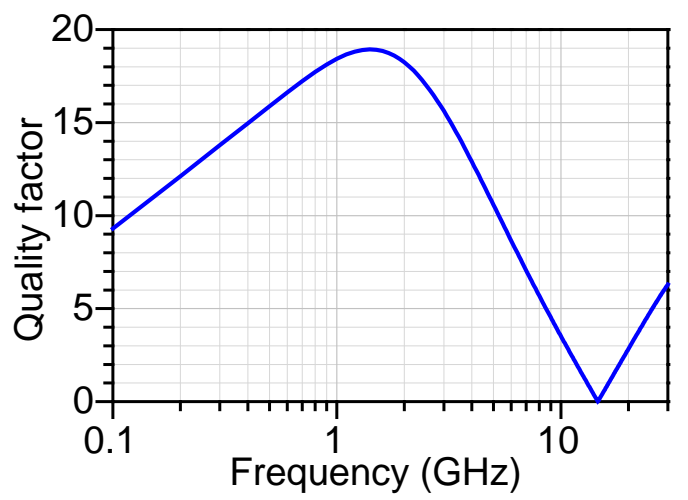

(b)

Fig. 5 Inductor performances in response of frequency variation (a) inductance value (b) quality factor. 
It is clearly seen at the Fig 5.a that resonant frequency is increased and reaches $15 \mathrm{GHz}$. In addition, the inductance value is amplified and it is comprised between $2.4 \mathrm{nH}$ and $6.5 \mathrm{nH}$. The quality factor is greatly ameliorated and reaches $\mathrm{Q}_{\max }=19$ at 1.5 GHz (Fig 5.b). We have investigated the new current distribution as shown in Fig.6:

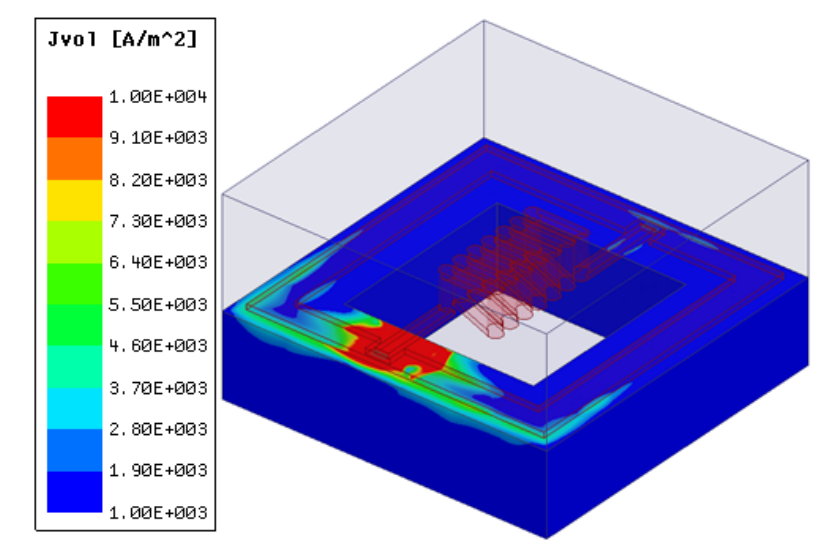

(a)

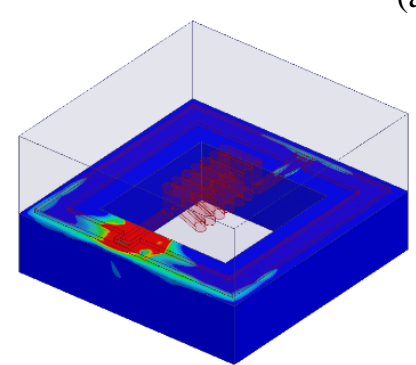

(b)

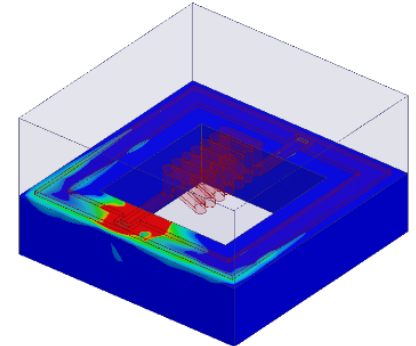

(d)

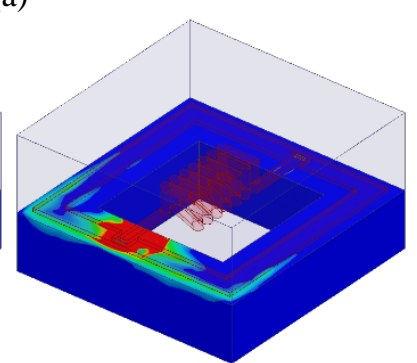

(c)

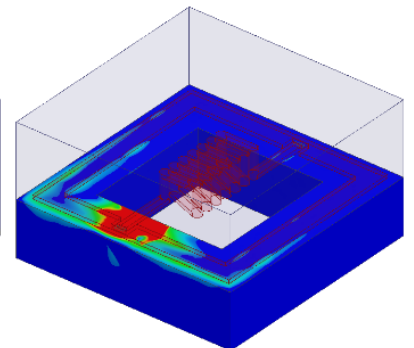

(e)

Fig. 6 Distribution of electrical current density in silicon substrate for different frequencies (a) aim frequency $5 \mathrm{GHz}$ (b) $100 \mathrm{MHz}$ (c) $10 \mathrm{GHz}$ (d) $20 \mathrm{GHz}$ (e) 30 GHz.

At $5 \mathrm{GHz}$ (Fig 6.a), the distributed current is localized at the excitation zone and it is almost negligible at the other parts of the substrate. Moreover, as we can see in Fig 6(b-e) the amount of the leakage current is became less sensible to the frequency variation. Indeed, the eddy current possesses almost the same repartition from $100 \mathrm{MHz}$ to $30 \mathrm{GHz}$. Consequently, we can conclude that the inductor performances are limited by the ability of lakage current penetration in silicon substrate.

\section{RF MEMS TUNABLE INDUCTORS}

\section{A. Current distribution with regular substrate}

The variation principle of our inductor is based on the modification of the solenoid core permeability in response of NiFe plate displacement. We have investigated the distribution of electrical current density on the ferromagnetic thin film for five positions. The Fig 7 illustrates the obtained results at $5 \mathrm{GHz}$ with $100 \mu \mathrm{m}$ successive step for each position: 


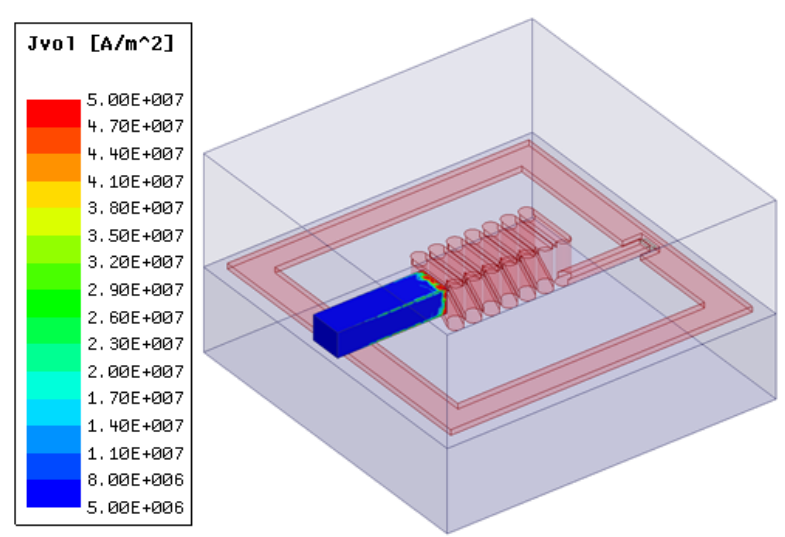

(a)

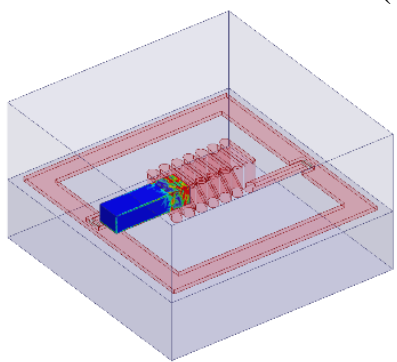

(b)

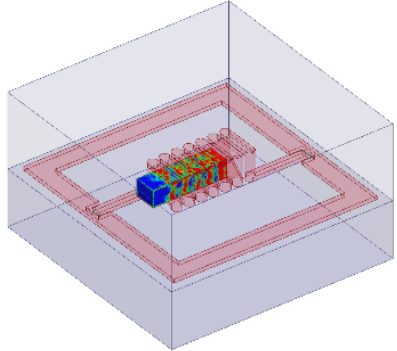

(d)

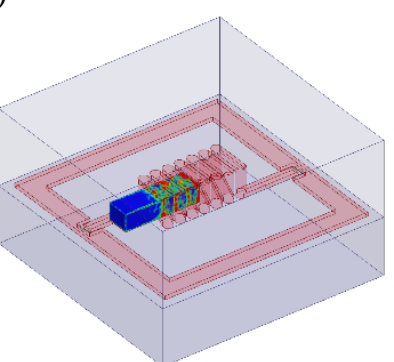

(c)

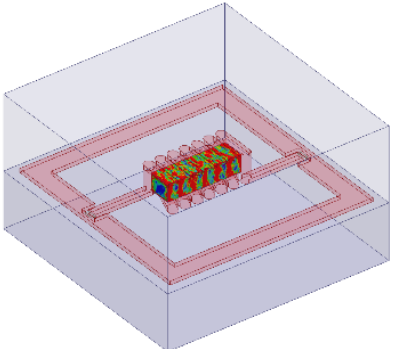

(e)

Fig. 7 Distribution of electrical current density in NiFe plate at 5 GHz (a) POS1 (b) POS2 (c) POS3 (d) POS4 (e) POS5.

As we can see in the Fig 7.a, a high level of distributed current appears in the head of the ferromagnetic thin film when it is placed at the front of the solenoid core. We have moved the NiFe plate by $100 \mu \mathrm{m}$ steps and we observed an increasing current density as illustrated in Fig 7(b-e). Indeed, the magnetic flux prevailing inner solenoid core induces electrical current in metal plate and the coupled magnetic field changes in response of the permeability of the ferromagnetic thin film.

\section{B. Performances variations with regular substrate}

Furthermore, in order to evaluate the effect of the NiFe plate displacement on the inductor performances we have drowned the quality factor and inductance value variations.

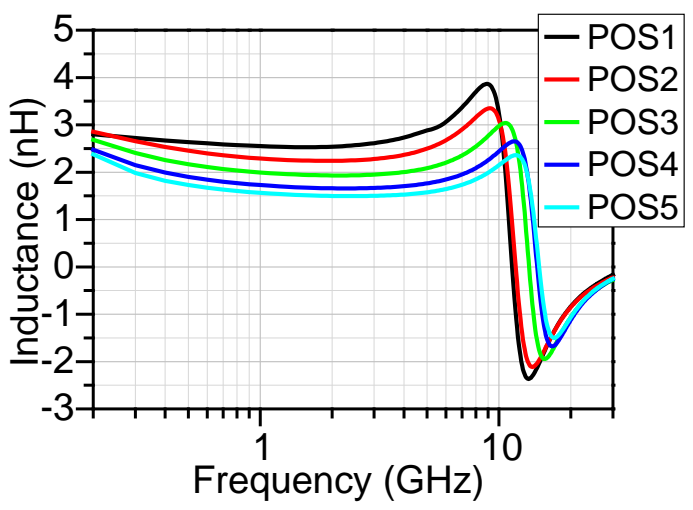

(a)

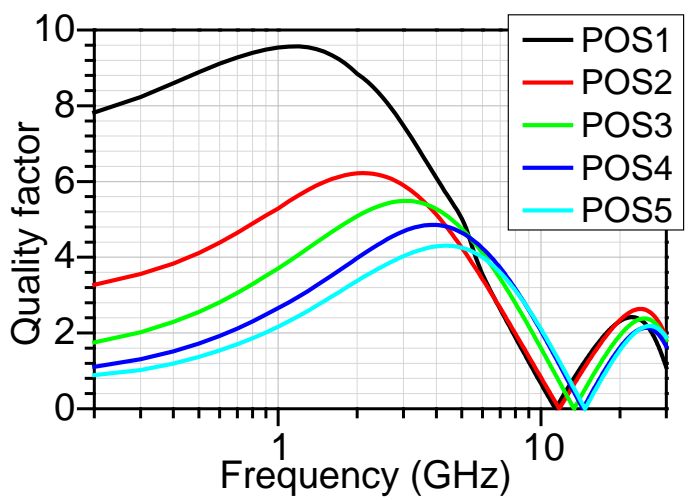

(b)

Fig. 8 Inductor performances in response of frequency variation for five positions of NiFe plate (a) inductance value (b) quality factor.

The Fig 8.a illustrates the inductance variation for the frequency range of $100 \mathrm{MHz}$ to $30 \mathrm{GHz}$. It is clearly seen that the inductance decreases in response of NiFe plate penetration in solenoid core. At $5 \mathrm{GHz}$, the inductor value is comprised between $\mathrm{L}=3.09 \mathrm{nH}$ without actuation to $\mathrm{L}=1.57 \mathrm{nH}$ when the $\mathrm{NiFe}$ reach the last position. Therefore, we obtain an importante inductance tuning range 
that reaches $\operatorname{Tr}=96.81 \%$. In addition, the quality factor fall down and became minimal $\mathrm{Q}$ min $=4.5$ when the ferromagnetic thin film occupy the whole solenoid core at $5 \mathrm{GHz}$.

\section{Current distribution with etched substrate}

We have used the same principle of variation with MEMS inductor in order to study the effect of etched silicon substrate on inductor performances. The Fig 9 shows the distribution of electrical current density on NiFe plate for five positions at $5 \mathrm{GHz}$ :

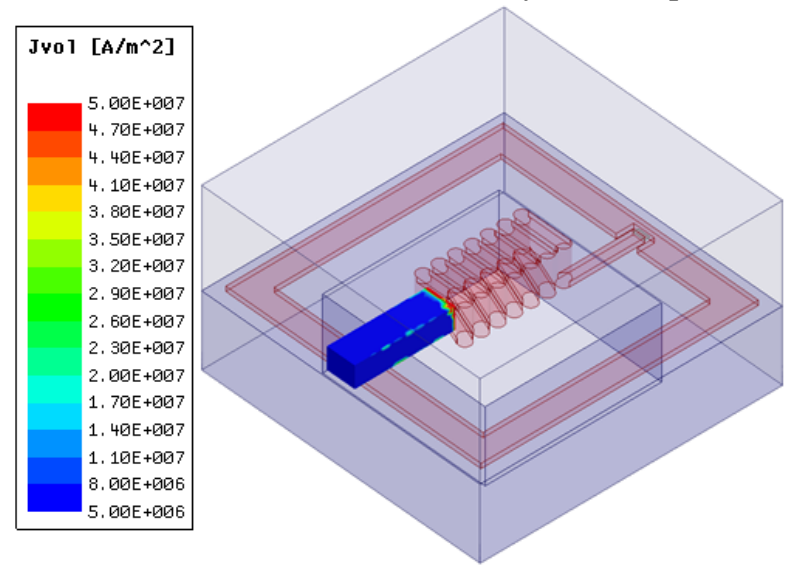

(a)

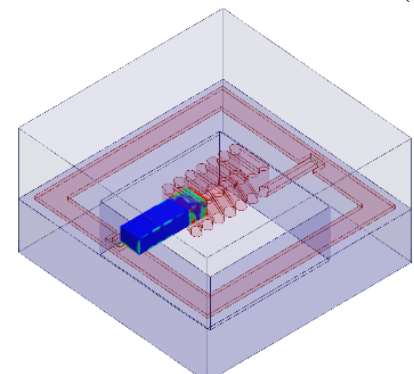

(b)

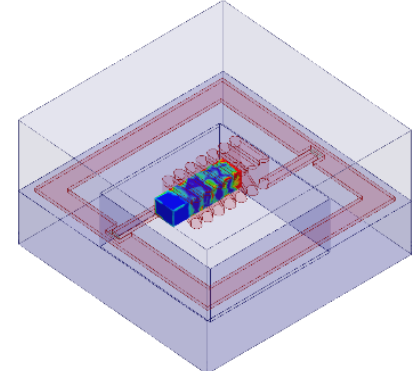

(d)

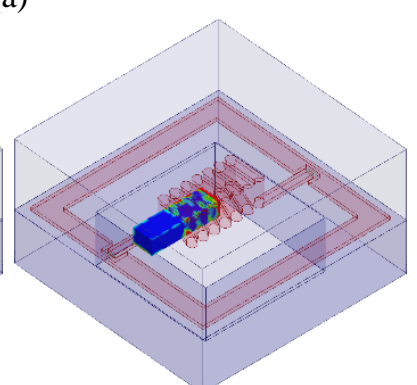

(c)

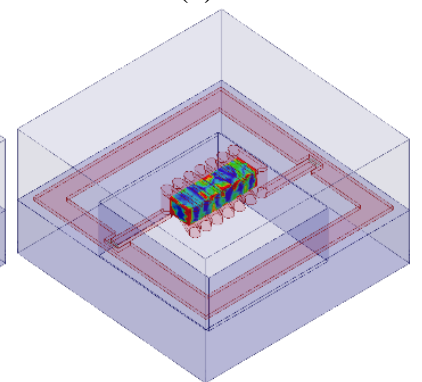

(e)

Fig. 9 Distribution of electrical current density in NiFe plate at 5 GHz (a) POS1 (b) POS2 (c) POS3 (d) POS4 (e) POS5.

For the first position of the NiFe plate (Fig 9.a), we observe almost the same current repartition as obtained in the previous study shown in Fig 7.a. Nevertheless, the current density clearly decreases compared to previous results when the ferromagnetic thin film penetrates the solenoid core. We can conclude that magnetic flux inner solenoid core is also influenced by the eddy current occurs in silicon substrate.

\section{Performances variations with etched substrate}

We have investigated the inductance and the quality factor variations for five NiFe plate positions. The Fig 10 illustrates the obtained results for frequency range from $100 \mathrm{MHz}$ to $30 \mathrm{GHz}$ : 


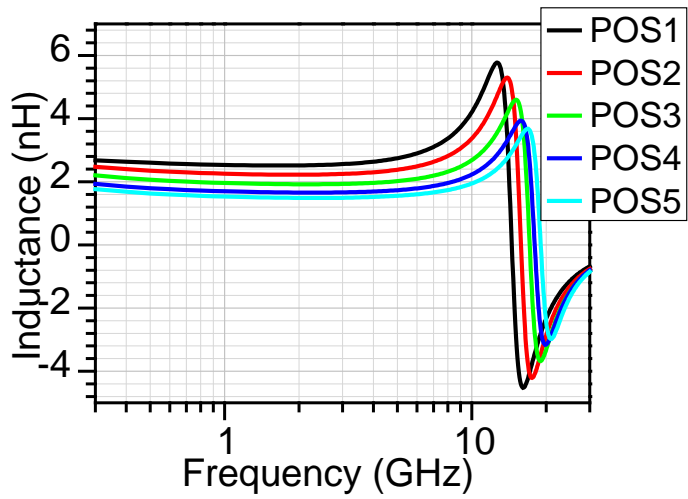

(a)

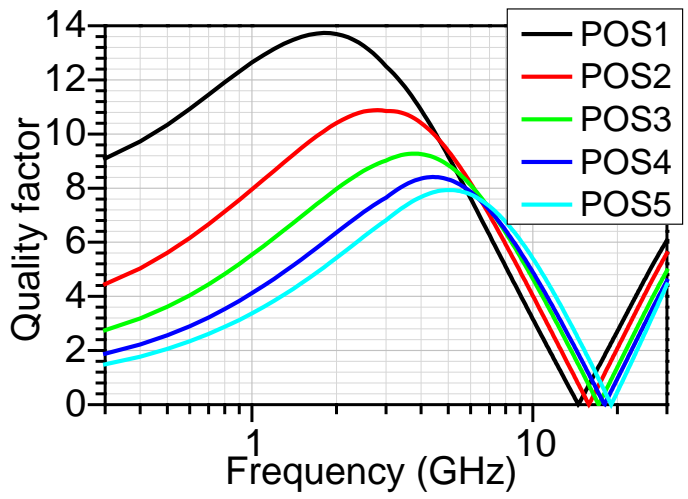

(b)

Fig. 10 Inductor performances in response of frequency variation for five positions of NiFe plate (a) inductance value (b) quality factor

The inductance curves illustrate a high resonant frequency accompanied with the diminution of inductor value in response of the ferromagnetic thin film displacement (Fig 10.a). The total inductance variation at $5 \mathrm{GHz}$ is comprised between $\mathrm{L}=2.9 \mathrm{nH}$ and $\mathrm{L}=$ $1.55 \mathrm{nH}$ that leads to $\mathrm{Tr}=87 \%$ and rise up to $\mathrm{Tr}=132.81 \%$ at $10 \mathrm{GHz}$. Moreover, the minimal quality factor value increases and reaches $\mathrm{Q}=8$ at $5 \mathrm{GHz}$.

\section{CONCLUSION}

Two different solenoid RF MEMS inductors are designed and studied: fixed and variable. In first case, we have investigated the current density distribution in silicon substrate and we have discussed the inductors performances in regular and etched silicon substrate. The obtained results show an important amelioration of quality factor and resonant frequency when silicon substrate is etched due to the diminution of substrate leakage current. In second case, we have discussed NiFe plate penetration inner the solenoid core in two substrates states. Once more, the performances of tunable inductors are also clearly ameliorated with etched substrate.

\section{REFERENCES}

[1] Banitorfian, F., Eshghabadi, F., Manaf, A. A., Pons, P., Noh, N. M., Mustaffa, M. T., \& Sidek, O. (2014). Evaluation and analysis of methods for fixed and variable MEMS inductors design. Inf. MIDEM, 44(2), 87-103.

[2] El Gmati, I., Calmon, P. F., Boukabache, A., Pons, P., Fulcrand, R., Pinon, S., ... \& Besbes, K. (2011). Fabrication and evaluation of an on-chip liquid microvariable inductor. Journal of micromechanics and microengineering, 21(2), 025018.

[3] Fukushige, T., Yokoyama, Y., Hata, S., Masu, K., \& Shimokohbe, A. (2003). Fabrication and evaluation of an on-chip micro-variable inductor. Microelectronic engineering, 67, 582-587.

[4] Lubecke, V. M., Barber, B., Chan, E., Lopez, D., Gross, M. E., \& Gammel, P. (2001). Self-assembling MEMS variable and fixed RF inductors. IEEE Transactions on Microwave Theory and Techniques, 49(11), 2093-2098.

[5] Habbachi, N., Boussetta, H., Boukabache, A., Kallala, M. A., Pons, P., \& Besbes, K. (2016). Tunable MEMS capacitor: influence of fluids. Electronics Letters.

[6] Pinon, S., Diedhiou, D. L., Gué, A. M., Fabre, N., Prigent, G., Conédéra, V., ... \& Boukabache, A. (2012). Development of a microsystem based on a microfluidic network to tune and reconfigure RF circuits. Journal of Micromechanics and Microengineering, 22(7), 074005.

[7] Habbachi, N., Boussetta, H., Boukabache, A., Kallala, M. A., Pons, P., \& Besbes, K. (2016). Fabrication and modeling of a capacitor microfluidically tuned by water. IEEE Electron Device Letters. 